\title{
Neurofibromatosis and small bowel adenocarcinoma: an unrecognised association
}

\author{
T J JONES AND T L MARSHALL \\ From the Department of Morbid Anatomy, The London Hospital Medical College, London, and the \\ Department of Histopathology, Central Pathology Laboratory, Stoke-on-Trent, Staffordshire
}

SUMMARY We present a review of the reported cases and describe a fifth of neurofibromatosis with small bowel adenocarcinoma and concurrent leiomyomas, indicating a previously unrecognised association.

Neurofibromatosis is known to be associated with an
increased incidence of neoplasms including phaeo-
chromocytoma gliomata and neurofibrosarcoma.'
Gastrointestinal involvement is well documented,
predominantly consisting of neurofibromas, ganglio-
neuromas and schwannomas. ${ }^{2}$ Epithelial tumours
including colonic, ${ }^{3}$ pancreatic ${ }^{4}$ and small intestinal
adenocarcinomas have all been reported. $\$-\$$

\section{Case report}

The patient, a 59 year old white man initially presented in July 1980 with a soft, well circumscribed mass $1.5 \mathrm{~cm}$ in diameter on the right thigh. This was excised and histology showed it to consist of spindle cells in a fibrillary stroma, appearances typical of a neurofibroma. On further clinical examination he was found to have a café-au-lait area on the left thigh; his father had been diagnosed as having neurofibromatosis in the past (no biopsy available). He was therefore diagnosed as a case of neurofibromatosis and remained asymptomatic for the next five years.

He next presented in February 1985 with intermittent colicky abdominal pain associated with vomiting, diarrhoea and $10 \mathrm{~kg}$ weight loss. On examination his abdomen was distended, with increased bowel sounds and tenderness over the right iliac fossa. Initial laboratory investigations showed a

Address for correspondence: Dr T J Jones, Dept of Morbid Anatomy. The London Hospital, London E1 1BB.

Received for publication 5 February 1987. neutrophil leucocytosis (WCC $19 \cdot 6 \times 10$ with $80 \%$ neutrophils). A diagnosis of small bowel obstruction was made and laparotomy revealed an area of stenosis in the middle third of the ileum with multiple serosal nodules. Thirty centimetres of ileum were resected and submitted for histological examination On opening the bowel an ulcerated stenosing lesion was noted $15 \mathrm{~cm}$ from the distal plane of excision along with multiple nodules on the rosal surface situated principally on the mesenteric srder.

Microscopy of the stenosing lesion showed it to be a moderately differentiated adenocarcinoma (Fig. 1) which had penetrated through to the serosal surface with metastases in the mesenteric lymph nodes (Fig. 2). The serosal nodules were shown to consist of spindle cells with cigar shaped nuclei arranged in interlacing bundles (Fig. 3); they arose from the muscularis propria and extended into the submucosa and onto the serosal surface. No neural elements were seen. Tissue from the nodules was placed in glutaraldehyde, postfixed in osmium tetroxide and examined using an AEI Corinth 500 Electron microscope. This showed intracellular filaments, adherent basement membrane like material and pinocytotic vacuoles. The overall appearances were of tumours of smooth muscle origin.

\section{Discussion}

Neurofibromatosis with involvement of the gastrointestinal tract is well documented ${ }^{\prime 2}$ and usually consists of neurofibromas, ganglioneuromas, and leiomyomas; much rarer is the development of 
Fig.1

Fig. 1 Section showing infiltrating adenocarcinoma. $H \& E . \times 50$

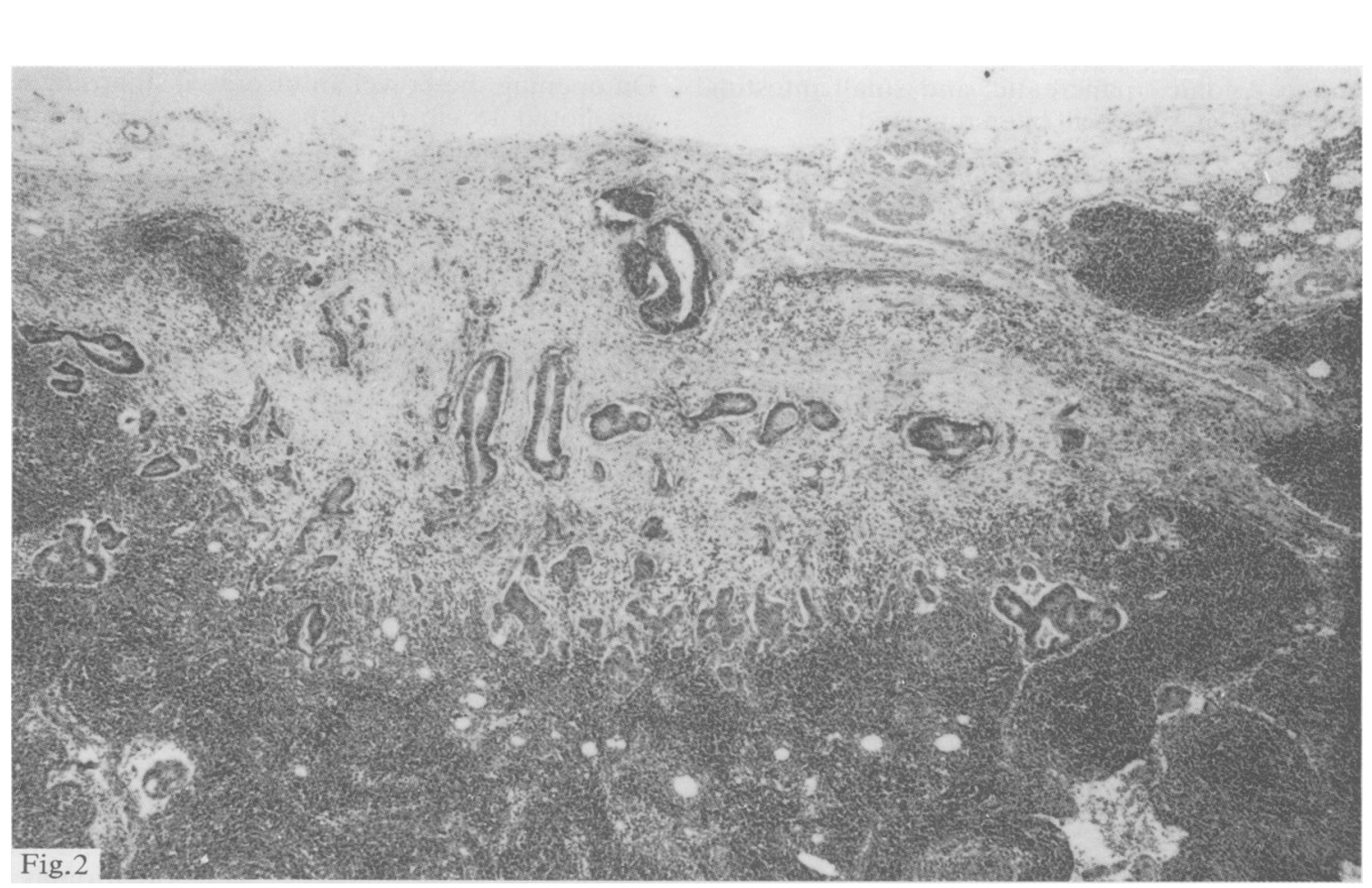

Fig. 2 Metastatic adenocarcinoma in subcapsular region of mesenteric lymph node. $H \& E . \times 50$

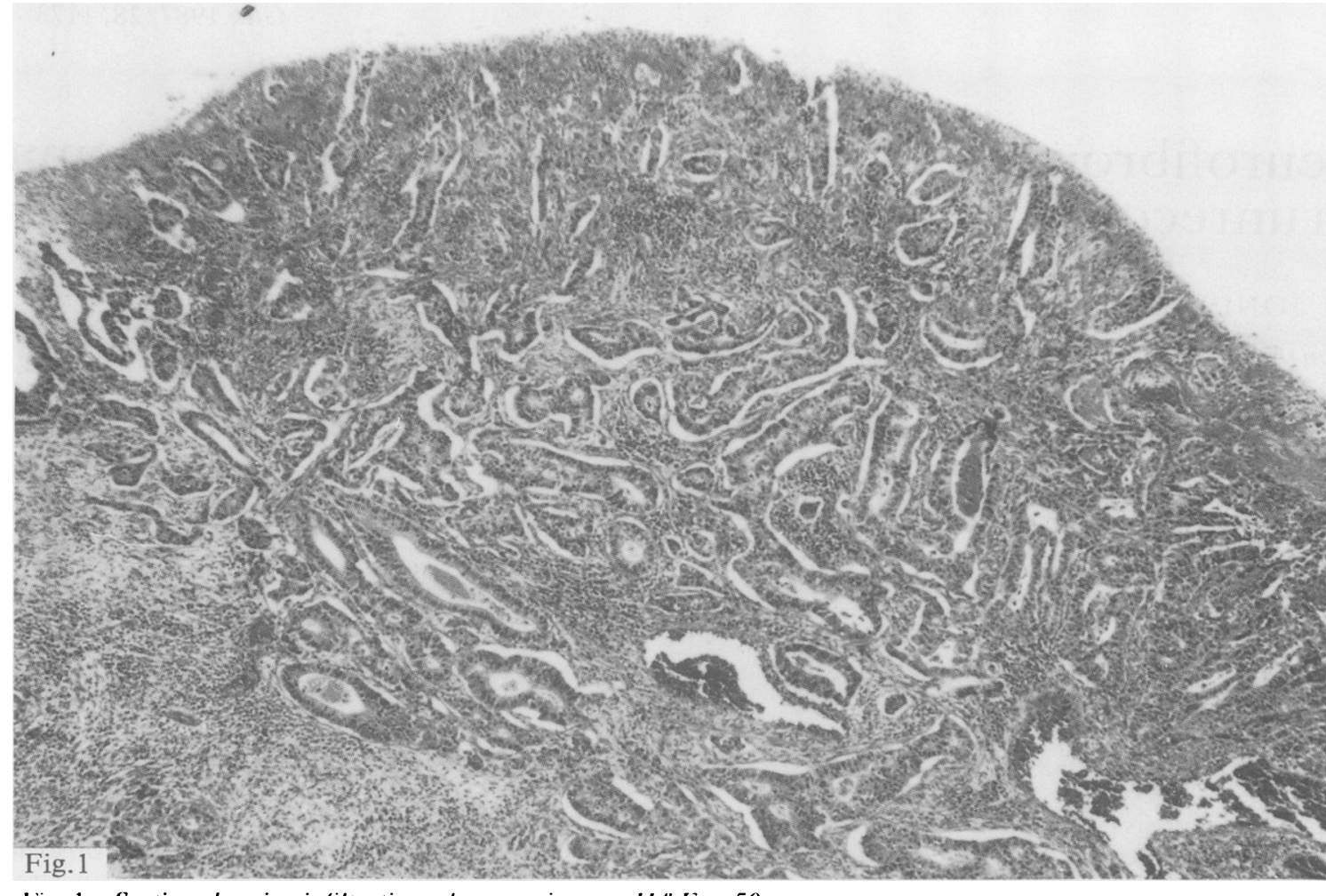




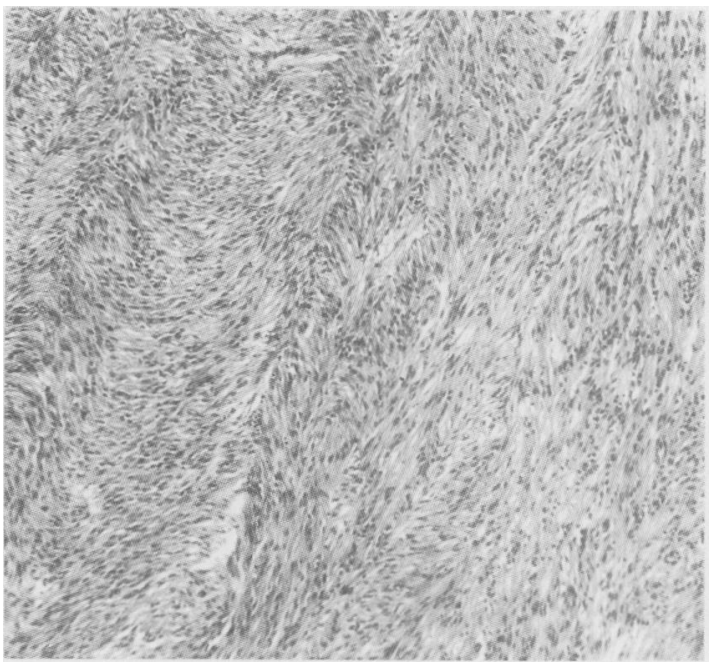

Fig. 3 Interlacing spindle cell pattern of serosal nodules. $H \& E$.

epithelial malignancy. Cases of adenocarcinoma of the colon ${ }^{3}$ and pancreas ${ }^{4}$ with neurofibromatosis have been described. In addition there have been four case reports of small bowel adenocarcinoma. ${ }^{-x}$ In three of these the neurofibromatosis was of the peripheral type $;-{ }^{-1-}$ in the remaining case the neural tumours were limited to the ileum and so were of the visceral type. ${ }^{x}$

The first case was reported in $1974^{\circ}$ in a 48 year old man who presented with small bowel obstruction and was found to have adenocarcinoma of the ileum with multiple nodules protruding from the mucosal surface. Microscopy showed these to consist of plexiform proliferation of neurites and Schwann cells interpreted as being plexiform neurofibromas. Multiple lymph node metastases were present. Cutaneous evidence of neurofibromatosis was not seen. The patient died in the immediate postoperative period.

The second case was reported in $1980^{\circ}$ in a 75 year old man who presented with anorexia, weight loss, and cutaneous neurofibromatosis. A large ulcerated, fungating tumour was present in the second part of the duodenum in the region of the ampulla. Biopsy of the tumour showed it to be a typical villous periampullary adenocarcinoma interpreted as arising from the duodenal mucosa. A laparotomy was carried out which showed multiple modules on the serosa of the jejunum and ileum which were interpreted as being metastatic deposits; a bypass procedure was done and the serosal nodules biopsied. Microscopy showed these to consist of spindle cell tumours interpreted as being leiomyomas. The patient died on the third day postoperatively.
The third case was reported in $1982^{\circ}$ in a 72 year old woman with cutaneous neurofibromatosis and weight loss. Small bowel radiography showed a polpoid mass in the ileum which was resected and shown to be a moderately differentiated adenocarcinoma admixed with a ganglioneuroma. Lymph node metastases were present. The patient was discharged one month after surgery.

The fourth case was also reported in $1982^{7}$ in a 29 year old man with jaundice, hepatomegaly and cutaneous neurofibromatosis. Laparotomy revealed a mass in the ampulla of Vater and a Whipples pancreaticoduodenectomy was carried out. Microscopy showed the mass to be a well differentiated adenocarcinoma with dysplastic changes in the surrounding duodenal mucosa and in the terminal common bile duct. Four lymph nodes contained metastatic carcinoma. The patient died four months postoperatively.

The above cases together with the one which is reported here indicate a previously unrecognised association between neurofibromatosis and adenocarcinoma of the small bowel including the periampullary region. Three "in of the reported cases also had intestinal stromal tumours: one with multiple leiomyomas, one ganglioneuroma, and one plexiform neurofibroma. In the latter this was the only manifestation of neurofibromatosis. The present case had multiple ileal leiomyomas. All the resected cases $^{t-x}$ including the present had local lymph node metastases at resection, indicating the usual advanced stage of small bowel adenocarcinoma at presentation. We believe that the association is unlikely to be caused by chance because of the very low incidence of small intestinal adenocarcinoma $(0 \cdot 5 / 100000$ population" and forming less than $1 \%$ of all intestinal neoplasms'"); the possibility of the two conditions occurring together spontaneously is therefore very slight.

\section{References}

1 Ghrist TD. Gastrointestinal involvement in neurofibromatosis. Arch Intern Med 1963; 112: 357-62.

2 Rutgeerts P, Hendrickx H, Gebdes K, Ponette E, Broeckaert L, Vantrappen G. Involvement of the upper digestive tract systemic neurofibromatosis. Gastrointest Endosc 1974; 27: 22-5.

3 Jenkins DHR, Gill W. A case of carcinoma of the colon in association with cutaneous neurofibromatosis. $\mathrm{Br} J$ Surg 1973; 59: 322-3.

4 Keller RT, Logan GM. Adenocarcinoma of the pancreas associated with neurofibromatosis. Cancer 1977: 39: 1264-6.

5 Ho KT, Tan KA, Gwee MM, Lee SK, Foong WC. A case of primary duodenal carcinoma and small bowel leiomyomas in a patient with neurofibromatosis. Singapore Med J 1980; 21: 713-6. 
6 Nelson AM. Small bowel adenocarcinoma associated with neurofibromatosis. Am J Gastroenterol 1982; 77: 149-51.

7 McGunchey JJ, Santer GJ, Haqqani MT. Primary adenocarcinoma of the duodenum associated with cutaneous neurofibromatosis (von Recklinghausens disease). Postgrad Med J 1982; 58: 115-6.

8 Albores-Saavedra J, Alcantra-Vazqueza A, Cruz-Ortiz
H, Olvera-Rabiela J, Rodriguez-Martinez A. Association of neurofibromatosis and adenocarcinoma of the small intestine. Patologia 1974; 12: 89-98.

9 Cancer Statistics Registrations. England and Wales; series MB1 No. 14. London: Office of population censuses and surveys, 1982.

10 Morson BC, Dawson IMP. Gastrointestinal pathology. Oxford: Blackwell, 1979: 408. 